\title{
Oncovascular Surgery; Surgery of the Vena Cava-Related Tumors
}

\author{
Kareem Sallam
}

One really wonders whether surgery is a branch of medicine or is it a branch of construction engineering

\subsection{Introduction}

The inferior vena cava, one of the most dreaded organs in abdominal surgery, is always blamed for killing subjects and more often is held the responsibility of the inoperability and irresectability of malignant disease.

Lying silently in the very depth of the abdominal cavity with many organs on its top, remaining patent despite its low pressure and unenforced wall.

The case for vena cava's implication in irresectability of tumors is similar to the usual scenario of living with human beings, the more you take responsibilities and commitments on your shoulder, the more you will be involved in issues of different kinds. The more you do, the more you will be blamed. This is the case with the vena cava, an organ with a very major role and numerous responsibilities and commitments to other organs with which it is connected to with tributaries many of which are critical.

As surgeons we commonly respond to this condition by abandoning or at least inconsistently doing surgery for tumors around the cava. While in fact all what it takes to perform surgery on an organ with multiple commitments is to be patient

\footnotetext{
K. Sallam $(\bowtie)$

Surgical Oncology Unit, Helwan University Hospitals, Cairo, Egypt

e-mail: kareem.sallam@med.helwan.edu.eg.
}

enough in the preparatory phase of surgery to repeat multiply the same step should the organ have had a single commitment.

If one would invest time to free the vena cava from its commitments and tributaries, he would find a very cooperative organ that would stand to his side and the side of the patient I assure.

So here comes the question: can all the vena cava-related tumors be resected?

With the use of current surgical and anesthesia facilities, perhaps the answer will be yes.

\subsection{Types and Different Views for Classifying Vena Cava- Related Tumors}

Vena cava-related tumors can have three different classifications (Table 32.1):

First is anatomical, i.e., according to the organ of origin.

Second is pathological, i.e., according to the histopathology of the tumor.

Third is technical (see later).

When you add the anatomy to the pathology, you can get numerous possibilities and combinations of anatomies-pathologies complexes.

The surprise carried by this chapter is that it is not about listing the vena cava-related tumors and then running through them to describe different operations for different tumors. Not at all. 
Table 32.1 Different ways to classify vena cava-related tumors

\begin{tabular}{l}
\hline 1. Anatomical, according to organ of origin: \\
\hline Posterior segment rt.lobe liver \\
\hline Caudate lobe liver \\
\hline Suprarenal gland \\
\hline Kidney \\
\hline Soft tissue of retroperitoneum \\
\hline 2. Histopathological: \\
\hline $\begin{array}{l}\text { Different types of carcinomas and sarcomas } \\
\text { according to the organ }\end{array}$ \\
\hline $\begin{array}{l}\text { Some benign and low-grade tumors of } \\
\text { retroperitoneal tissues and organs }\end{array}$ \\
\hline 3. Technical: \\
\hline 1. Tumors adjacent to the cava or encasing without \\
infiltration \\
\hline 2. Tumors infiltrating the full thickness of the wall \\
\hline 3. Tumors inside the caval lumen
\end{tabular}

This article is about introducing, as the title described, the concept of vena cava-related tumors all in one and encompassed as a single entity. The chapter's purpose is to describe the basic principles and the logic behind a single unified approach for what the author sees as a single entity from the technical standpoint unifying a variety of anatomies and pathologies when it comes to resection, an approach that enables getting to terms with the vena cava and may be with every retroperitoneal resection surgery.

Technically speaking, IVC-related tumors can be looked at as of three types:

Tumors adjacent to the cava without infiltration

Tumors infiltrating the caval wall

Tumors inside the cava

\subsubsection{General strategy of resection (the 4 phases of the surgery)}

The approach entails four main phases. The titles of which are given in Table 32.2 and the description follows.

There will be no surgery for any retroperitoneal structure (which is the case for the cava) without the more superficial organs regardless their name, size, and relevance being moved out of the way. This is basic and final. The problem is that sometimes the size of the tumor overshadows the organs and attracts the surgeons' atten-
Table 32.2 The four main phases for resection of vena cava-related tumor

Look at the scans and define the circle of interest, i.e., field of surgery (see text for description)

Phase 1: Mobilization of organs from in front of the circle of interest

Phase 2: Control of "ins and outs" to and from the circle of interest defined and now cleared from superficial structures by virtue of phase one

Phase 3: Complete (lateral and posterior) mobilization of the tumor-cava complex

\begin{tabular}{l}
\hline Phase 4: Three options \\
\hline 1. Subadventitial dissection of the cava \\
\hline $\begin{array}{l}\text { 2. Resection of cava with the tumor and } \\
\text { replacement as appropriate }\end{array}$ \\
$\begin{array}{l}\text { 3. Opening the cava to excise a tumor from within } \\
\text { the lumen }\end{array}$ \\
\hline
\end{tabular}

tion as soon as the abdomen is open, and therefore the surgeon goes straight to the visible and palpable part of the tumor disregarding the invisible part and disregarding the fact that he is only palpating the tumor through the full thickness of other organ, for instance, a thinned out mesentery of small bowl and colon. The anatomy will stay a fact; no matter how big the tumor is, this will never get the organs and their attachments to disappear. They might just be overshadowed or change the very usual position, but they will indeed remain in the abdomen.

The general configuration of organs in the abdomen is that we have two central structures which offer blood supply-namely, IVC and aortawhile the rest of the organs are on either sides of such central structures or on top of it. Whether on the sides or on top, they need to connect to the central structures by branches which will run in an axial plane for organs on the sides and in a sagittal plane for organs on top (superficial).

The organs in the abdomen lie in specific locations. Two categories of attachments keep them in position. First is their "ins and outs," namely, blood vessels and ducts, if any. These are unnegotiable; there interruption entails either resection of the organ or the need for a restorative surgery in most of the occasions.

What is dispensable in almost all abdominal organs is the second category which is the ligamentous attachments which in fact are nothing but perfectly dispensable peritoneal folds. 
One would be amazed how much mobility that the organs acquire as a consequence to the complete division of these almost bloodless and routinely accessible peritoneal reflections. A degree of mobility that makes the need to divide any of the first category attachments unnecessary in almost all surgeries of the abdomen. When these cuts are made as complete as they can get, every organ on the right-hand side can go to the extreme left and vice versa and sometimes can be even flipped cranially or caudally. In a sense the blood supply will act as a fulcrum with the organ at its distal end having a 3D mobility in different directions and hence exposing the central structures in the retroperitoneum.

The ability and the will for mobilizing whatever necessary of the abdominal organs is the key to accessing the vena cava.

Access solves almost all problems in surgery because otherwise one would do nothing but making a cut or placing a stitch or multiples of the two. Making a cut and placing a stitch is something that all surgeons do with reasonable ease and efficiency on the skin level. The only difference between skin and retroperitoneal surgery (including the vena cava-related tumors) is the access.

Here comes the list of organs that is on the way to the vena cava if the full length of the vena cava is to be exposed (Fig. 32.1):

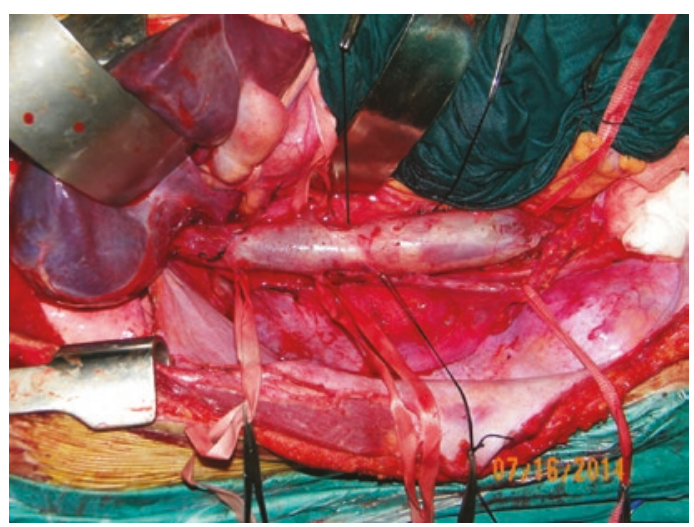

Fig. 32.1 The full length of vena cava exposed from diaphragm down to iliac bifurcation. Note the clearance of left side of the intrahepatic vena cava off the caudate lobe of liver. The free space here allows the passage of a big side clamp that controls the confluence of hepatic veins to cava as shown in Fig. 32.2a
- From top to bottom: Liver, Rt. colon, duodenum- head of pancreas and small bowel. (The last 3 are in a sense a complex since the fulcrum of the tree is the Superior mesenteric vessel).

Mobilizing the liver entails cutting the falciform (and round ligament), rt. triangular ligament, coronary ligament (and rt. triangular ligament)

The tricky part of freeing the cava from the liver is in freeing the short hepatic veins and clearing the left side of the upper cava from its intimate relation to the caudate lobe in particular. This maneuver is seriously important as it (1) prevents back bleeds from these veins, (2) enables placing a clamp on the joint venture of the hepatic veins preventing back bleeding even if the cava is opened straight away to the atrium as is required in some surgeries (Fig. 32.2), and (3) gives the cava this desired complete freedom and mobility facilitating opening, repairs, and resection and replacement should this be chosen for any reason.

During mobilization of the cava at this segment, it is to be appreciated and taken advantage of that the cava in its retrohepatic and intrahepatic segments is completely devoid of tributaries to its posterior surface. All what is to be handled with care and patience is the anterior and anterolaterally placed tributaries (short hepatic veins). Otherwise posterior mobilization can be done strictly by blunt finger dissection passed with relative impunity underneath the cava between it and the crus of the diaphragm. It is to be noted however that blunt finger dissection is not a careless forceful dissection. It is still to be done with gentleness. To the maximum, this can be upgraded to "conscious firmness" concentrating on the tactile feedback and stopping as resistance is encountered. When this resistance is met, the surgeon can retract the part that has been mobilized, sit on a stool to have a look behind the cava to see, and handle whatever is stopping the blunt dissection. Figure 32.2 shows options for hepatic veins control. Table 32.3 contains notes on hepatic veins control.

On mobilizing the rt. colon-small bowel complex, the surgeon needs to pay attention to cut the root of the mesentery of the small bowel and not to settle only to evisceration of the bowl. The root of the mesentery crosses obliquely in front of the 

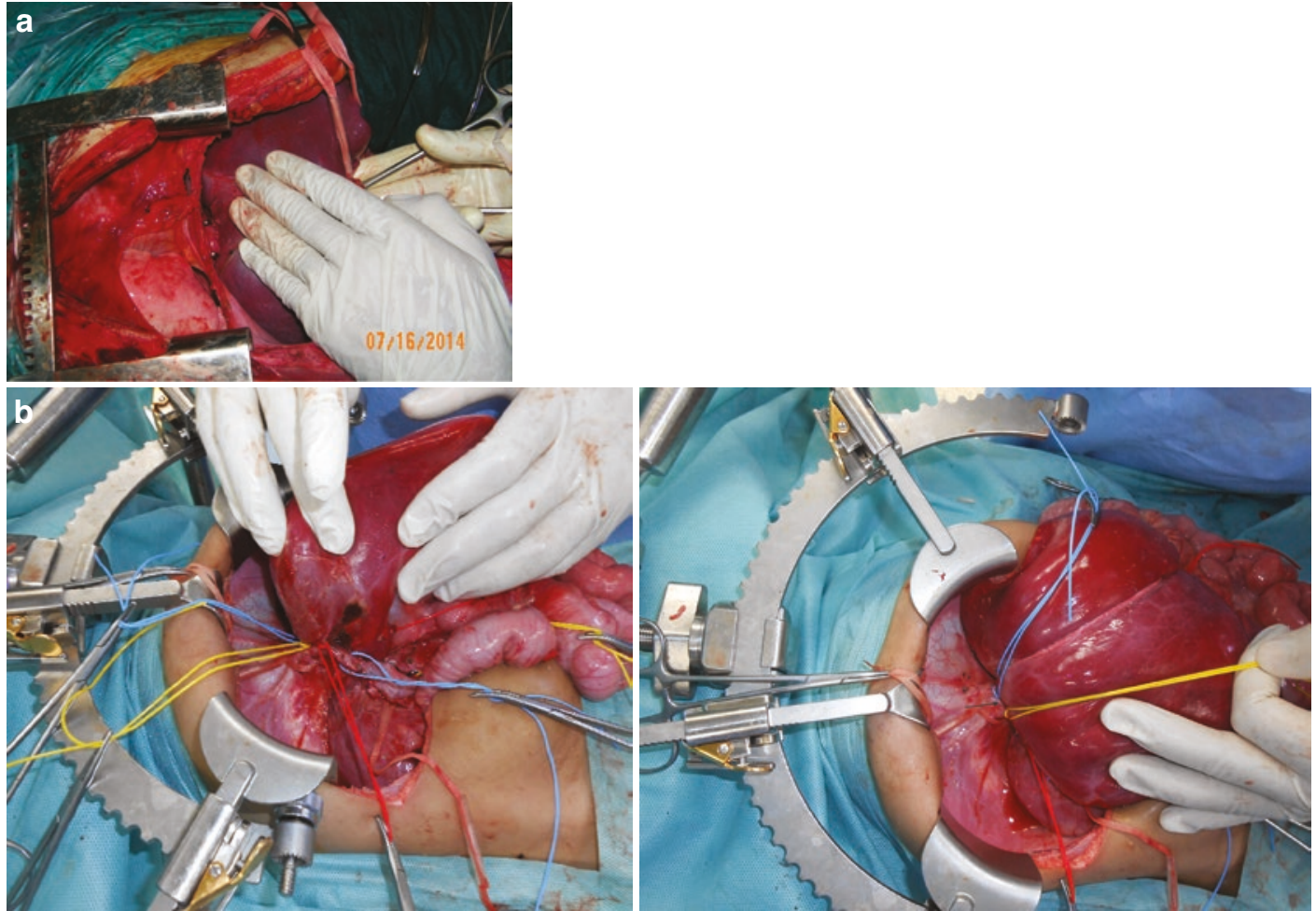

Fig. 32.2 Options for hepatic veins control. (a) Common control; A Satinsky clamp controlling the hepatic veins entry to cava in totality. (b) Selective control; Selective control by rubber slings. Please see text for description

Table 32.3 Notes on hepatic veins control

Notes on hepatic veins control

1. In either A or B, hepatic veins control is not to be attempted before complete freedom of intrahepatic cava is achieved by ligation of short hepatic veins from all directions. The hepatic vein stumps gain much length when this is done combined by outward traction on the liver. Care is to be taken not to misidentify the main hepatic veins as a large short hepatic vein

2. Main hepatic veins are wide based, and therefore standard looping using a right angled forceps is usually not possible if not hazardous; the rt. angled forceps' blade is never long enough to cover the whole width of them. The technique used by the author is as follows:

-Complete freeing of the cava from short hepatic veins

-An artery forceps has its back lying flat on the midline of the cava (i.e., the tip is toward the ceiling) from the caudal side and from the cranial side alternatively until it shows up from the other direction. This necessitates observing a characteristic notch that appears on the caval wall at the central tendon side demarcating the boundary between the rt. and the middle hepatic vein marked by an arrow in Fig. 32.2b lt. This notch guides the placement and direction of the aforementioned artery forceps. As the artery forceps aims at coming out from the other side, its tip should stay directed to the ceiling till showing up again after traversing the full length of the hepatic vein entry to cava. This avoids faulty outwarding through the hepatic vein wall itself which is thin and compressible and might give the operator an impression that it is a connective tissue band specially when flattened against the artery forceps or indeed the rt. angled forceps (Fig. 32.3)

3. Lt. hepatic vein isolation requires working from the left-hand side and division of the lesser omentum in order to gain access to the area of concern (Fig. 32.4). It might require as well clearing off hepatic parenchyma that usually covers part of the lt. hepatic vein entry to cava 


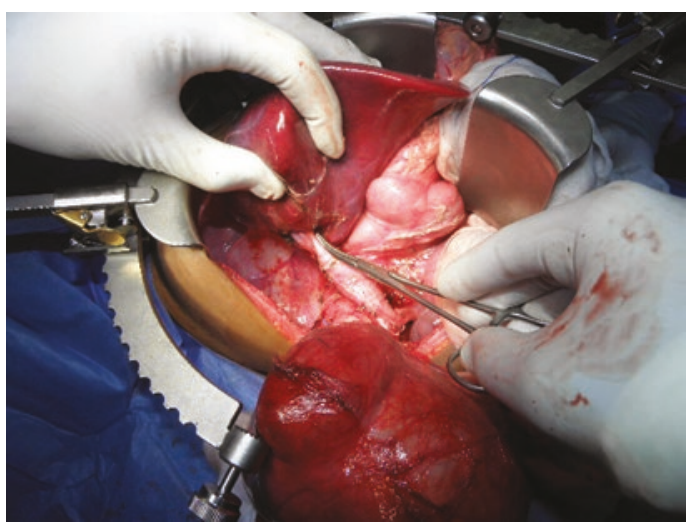

Fig. 32.3 Slinging the rt. hepatic vein: the use of an artery forceps and the direction of its introduction

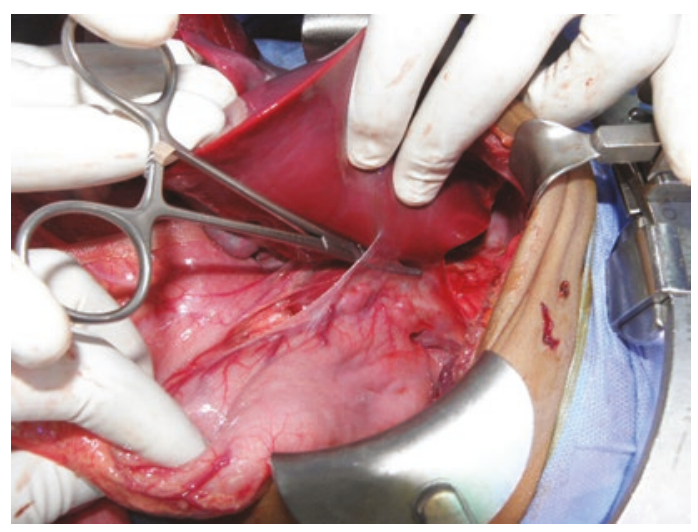

Fig. 32.4 Exposure of lt. hepatic vein from lt. side: division of the lesser omentum gives access to cava from 1 t. side

retroperitoneal central structures (Fig. 32.5). Cutting this flimsy attachment maximizes exposure and enables flipping of the bowel cranially toward the patient's chest. A maneuver helps much in access and in freeing the hands of the assistants (Fig. 32.6).

Cranial as opposed to lateral flipping of small bowel is to be used when exposure of the aorta and cava is required to take place at the same time and in the same field. In these occasions, cranial flipping might be better (Fig. 32.6).

Table 32.4 describes the different options for small bowel mobilization according to the required magnitude of exposure of the retroperitoneum.

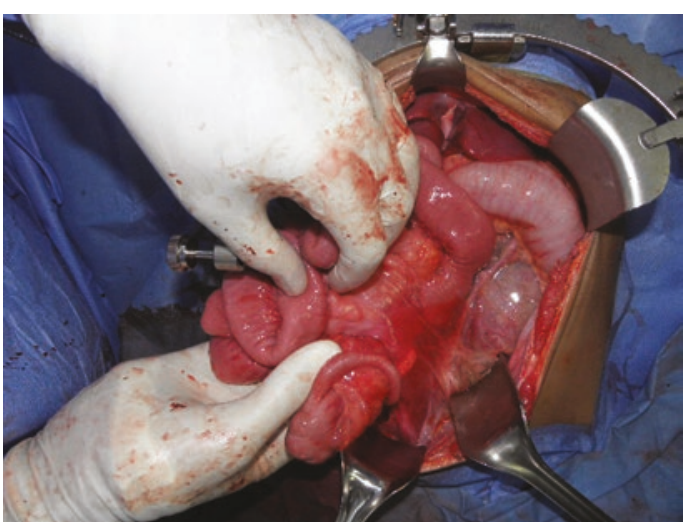

Fig. 32.5 The root of the small bowel mesentery; note that the small bowel is already eviscerated and mobilized laterally. Releasing the root of the small bowel mesentery enables flipping the bowel cranially and not only laterally maximizing the exposure of the vena cava and its tributaries and indeed the related tumor as shown in Fig. 32.1

Though mobilizing the organs is the first manual step, it is preceded by a mental one, a step that the author describes as step zero. This step is done on the scans in which the surgeon, with eye on the scans, defines the circle of interest which is the field of surgery, the field that needs to be cleared to start dissection of the tumor. This circle will be the surgeon's world for the surgery of resection, but one has to define it in the first place, and the advice here is to delineate a circle that encompasses the tumor range and a "bit more" in every direction.

This "bit more" is a rim of anatomical field all around the tumor in which the surgeon will patrol looking for blood vessels and ducts getting in and out of the field of the tumor to identify and sling. On top of which undoubtedly is the proximal and distal control of the vena cava. This step of patrolling and slinging comes as the second phase of the surgery after the organ mobilization phase. When completed, and only when it is, the surgeon can now "recall" that he is here to resect a tumor and pay attention to the tumor-vena cava complex.

Now the surgeon has the cava adherent to the tumor and the tumor adherent to the retroperitoneal structures and posterior abdominal wall, so where to start? 

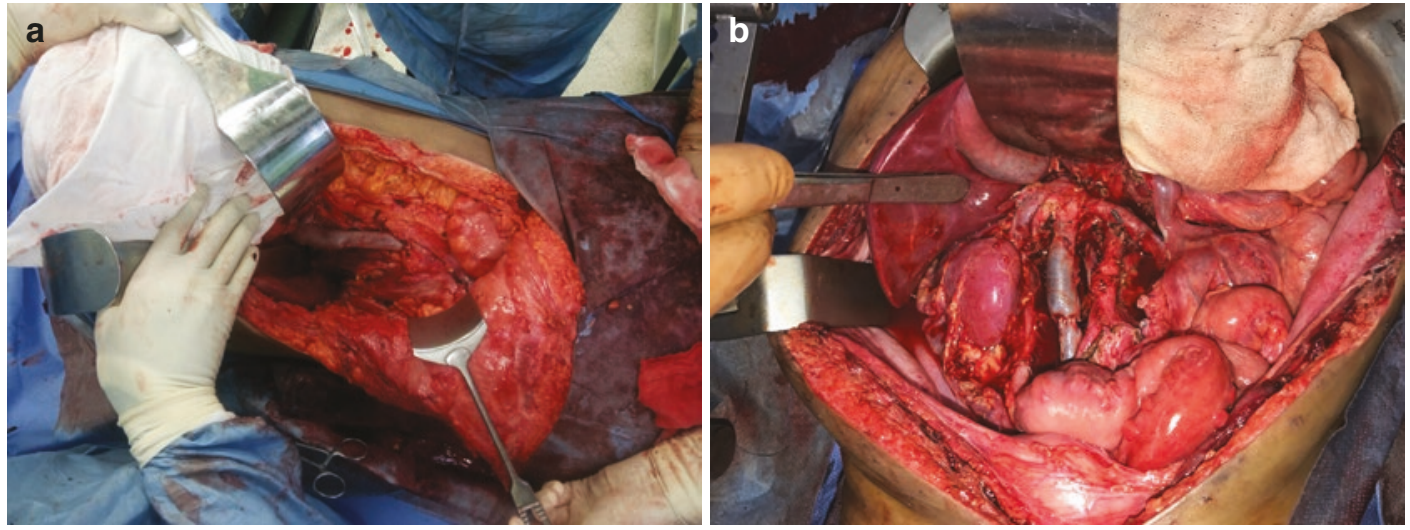

Fig. 32.6 (a) Cranial flipping of small bowel. (b) Another example of cranial flipping of small bowel wrapped in white towels. This maneuver exposes both cava and aorta in the same field

Table 32.4 Options for small bowel

Options for small bowel

This is a ladder of options according to degree of exposure required

\section{Packing}

\section{Evisceration}

3. Tenting: In which root of mesentery released yet coecum stays in right iliac fossa, and the surgery takes place underneath the small bowel and rt. colon mesentery (Fig. 32.7)

4. Complete mobilization: Root of mesentery released and coecum released completely as well leading to "mobile evisceration" of whole small bowel and rt. colon (Figs. 32.5 and 32.6)

It is usually tempting to many surgeons to go straight to the cava to take off the tumor and breathe the sigh of relief and as well decide "resectability," for some will abandon the procedure if the vena cava is inseparable from the lesion.

The answer to the question "where to start?" is fairly similar to the situation of examining the abdomen in clinical rounds. You ask where the pain is and you start at the farthest point palpating the abdomen leaving the quadrant of pain till the end.

The tumor is to be mobilized laterally first with complete disregard to the cava. Only when the tumor and cava are mobilized as one complex one can start handling the cava, and this marks the fourth and final phase of the surgery, the phase of handling the cava.

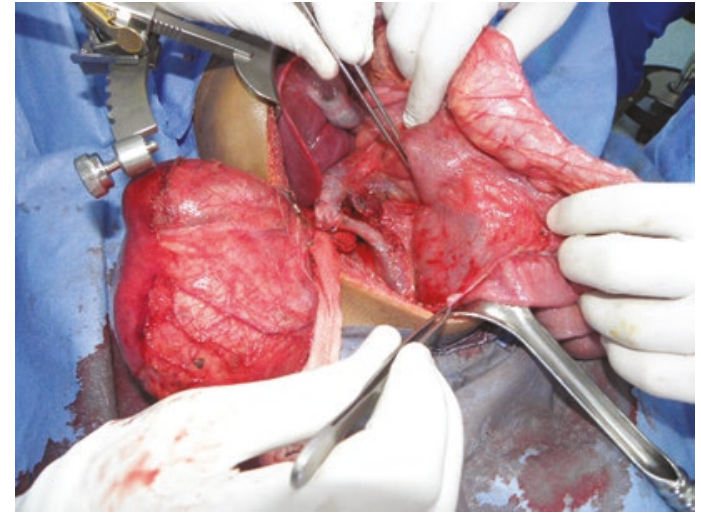

Fig. 32.7 Tenting of the small bowl mesentery and working from underneath. The coecum can stay in place in the rt. iliac fossa or acquire partial mobilization

This is the variable step of the procedure and the options are (Fig. 32.8):

1. Subadventitial dissection of the cava off the tumor

2. Resection of the tumor-cava complex and replacement by a graft

3. Opening the cava to excise a tumor thrombus

Even for surgeons who are making a decision to abandon the procedure if the cava is inseparable from the tumor, the author would recommend leaving the cava till the end. One will be amazed how friendly is this badly reputed organ will be when the procedure is done in this order. The surgical 

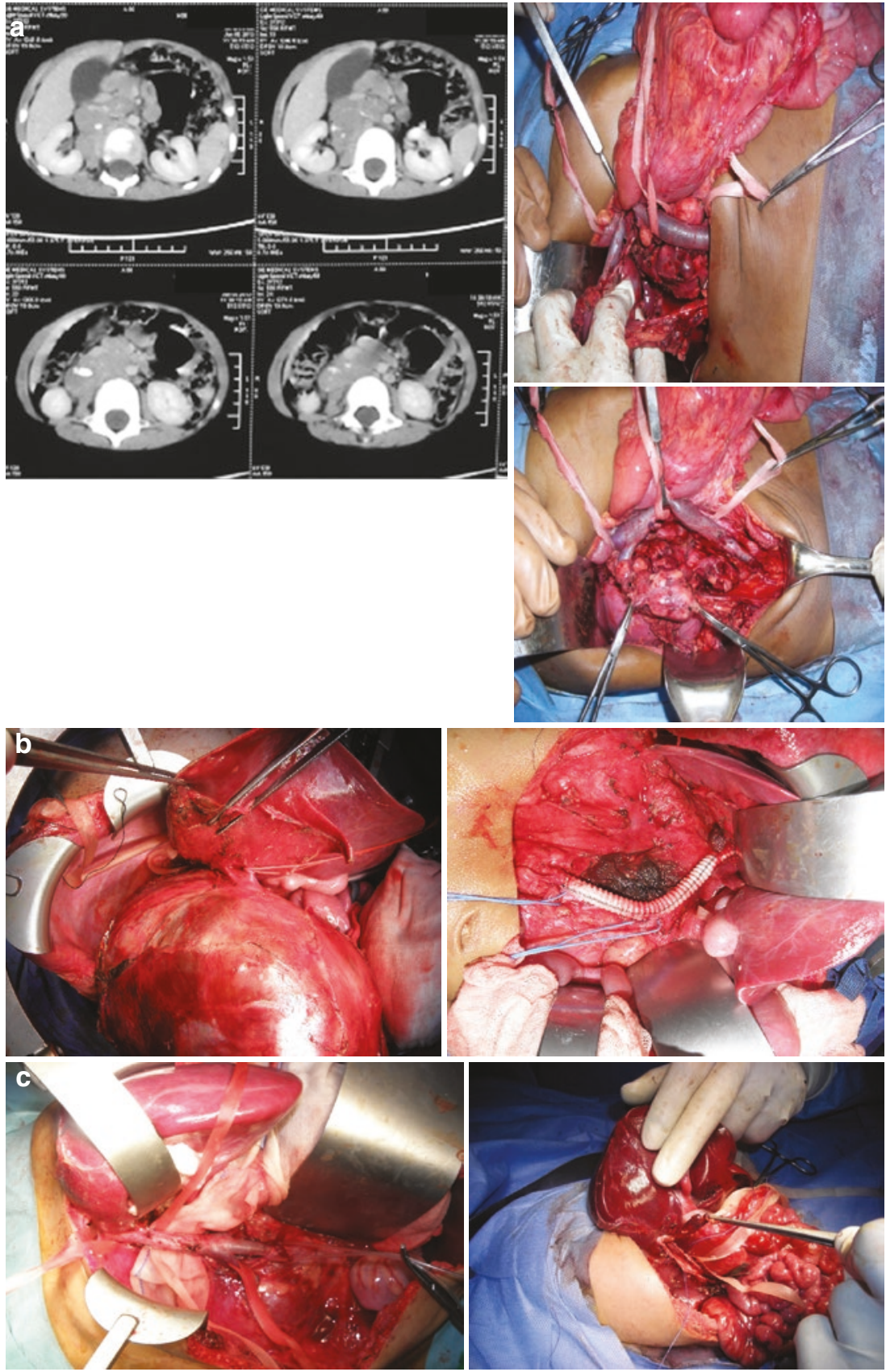

Fig. 32.8 The three options of phase 4 in surgery of vena cava-related tumors; (a) Subadventitial dissection, a patient with centrally located neuroblastoma: demonstration of the use of slings to retract the cava in different directions and perform subadventitial dissection. (b) Resection and replacement: a patient with retroperitoneal recurrent soft tissue sarcoma status postresection and rt. nephrectomy. Now with recurrence encasing and infiltrating the IVC and part of the lt. renal vein caudal surface. Resection was performed with replacement of the IVC by Dacron graft and patch graft on the renal vein with internal jugular vein graft. The patient underwent uneventful recovery and discharged on therapeutic anticoagulation. (c) Extraction of a tumor from within the caval lumen. Lt.: complete exposure of cava by mobilization of bowel and liver, proximal and distal control as well as control of tributaries. Complete freeing of the cava as demonstrated by the small swab passing freely round the cava. Rt.: now the cava can be opened and the thrombus dissected off the wall by a variety of instruments including endarterectomy dissector. Note the en bloc excision of the thrombus 
team will find a cooperative organ tolerating much traction, dissection, and repairs when needed, all enabling dissection of the cava that would, in other wise order, have seemed inseparable from the tumor.

The technique of dissection of the vena cava off the tumor entails using No. 15 blade lying flat. As soon as a window can be opened anywhere along the caval segment in question, a sling is to be passed round the cava and pulled in different directions including upward. This sling retraction enables maneuvering the cava here and there giving access to its different facets including the posterior one, and hence dissection can proceed and the short-stumped tributaries viewed and get a bit of length for control and division.

The sling belongs to the main operator's dominant hand with a dissection tool in the other being it a blade, a scissors, a dissection forceps, a sponge on a stick, or a Poole Suction Tip with the shield removed. Poole Suction Tips with holes very close to the tip are favored when suction tips are used for dissection. The proximity of holes to the tip makes them suck minute amount of blood rapidly and hence clearing the field for dissection. For scissors, a standard Metzenbaum is one friendly instrument. It is of value to add to the set a round-tipped Reynolds scissors of variable lengths.

An experienced assistant would have forceps in his non-dominant hand and suction tip in the other if the main operator is not holding it. Otherwise another forceps or counter traction by a small swab should be the case. The suction in whoever's hand is not only for sucking but also for teasing the tissues trying to open up the plane.

The case for vena cava dissection is the same for the dissection of any vessel; the more you go close to its wall, the easier is and the safer is the plane and not otherwise. That is why it is called subadventitial dissection. The cava lies within a sheath. The first step in dissection is opening up this sheath longitudinally along the vena caval segment in question. It is as if the cava is putting on a shirt with buttons on the front. Desheathing the cava is like taking off a shirt off a man's body, you release the buttons from the front surface and the rest of the sheath will then retract to either side (Fig. 32.9).

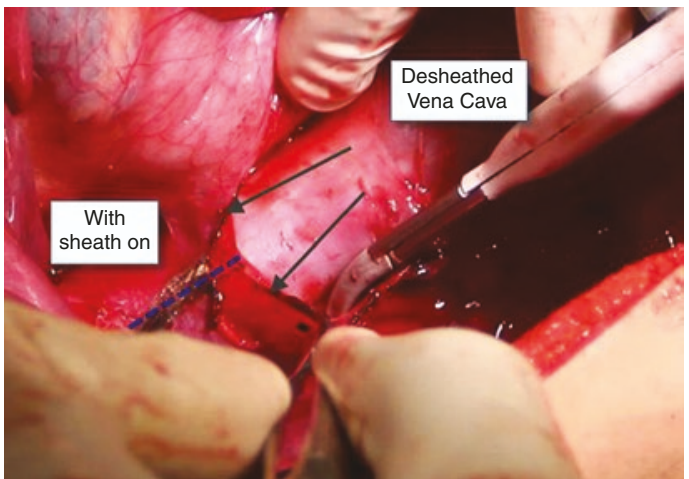

Fig. 32.9 Desheathing of the cava in progress. The view obtained in figure one with all tributaries looped around is facilitated by desheathing of the cava (see text for description). The cut edge of the sheath is pointed to with black arrows with a vena caval segment. When the sheath is cut on the strict front side of the cava longitudinally, this allows the side sheath to retract laterally; with the aid of a blunt instrument, the desheathing is completed in the opened plan. In this photograph a bipolar biclamp cautery instrument (LigaSure, precise handle by Covidien) is being in use. The dotted line represents the presumed direction of initial cut to be made by the cautery instrument

\subsection{Remarks, Adjunctive Techniques, and Special Situations}

\subsubsection{Remarks}

This section draws some light on specific points in the approach abovementioned. The points chosen are presented in a chronological sequence as they happen in surgery.

- Tumor embolism and the use of preoperative IVC filters

- Positioning of the patient

- Incision

- Role of table-mounted retraction and magnification

- The difference between lateral dissection and posterior dissection

- Vena cava clamping

- Vena cava grafting

- Inviting an old technique: vena cava compartmentalization 


\subsubsection{Tumor Embolism and the Use of Preoperative IVC Filters}

Particularly for the case of intraluminal tumor thrombi, the risk of embolism is of concern. Early proximal clamp is always advisable. The use of preoperative IVC filters is another option. These are better to be retrievable, and there needs to be a space of at least $4 \mathrm{~cm}$ before the cavo-atrial junction to enable deployment of the filter. Transfemoral access routes are strictly prohibited for the risk of embolism as a result of manipulation of the thrombus by the passing endovascular tools.

The authors have dealt with four types of intraluminal tumors in the cava and some other veins, namely, Wilm's tumor, sarcomas of different kinds, renal cell carcinomas, and thyroid carcinoma.

Out of the four, the renal cell carcinoma has been found to be very liable to detachment, and hence early proximal clamping is mandatory. The other three has been found friendlier. Particularly with Wilm's tumor, thrombi tend to be adherent to the wall by synechia which probably arise after chemotherapy. Sarcomas have been found to be firm and compact and rubbery. The initial touch of the thrombus informs about its firmness, attachment to the wall, and coherence. This should indicate the need for early proximal clamping.

\subsubsection{Positioning of the Patient}

A roll underneath the site of surgery is the standard for abdominal surgery. When surgery includes resection of a tumor, surgeons tend to put the roll underneath the equator of the tumor. The recommendation here is, again, to ignore the main bulk of the tumor and its equator. The roll is best put underneath the part of surgery that is farthest or critical in access. In the case of vena cava-related tumors, this is usually the upper pole of the field of surgery rather than the equator of the tumor. This is the point of proximal control.

\subsubsection{Incision (Fig. 32.10)}

Options are:

\section{Bilateral Transverse with Supraumbilical Midline Extension}

It gives very good vertical access as well as horizontal one where the surgeon can approach the

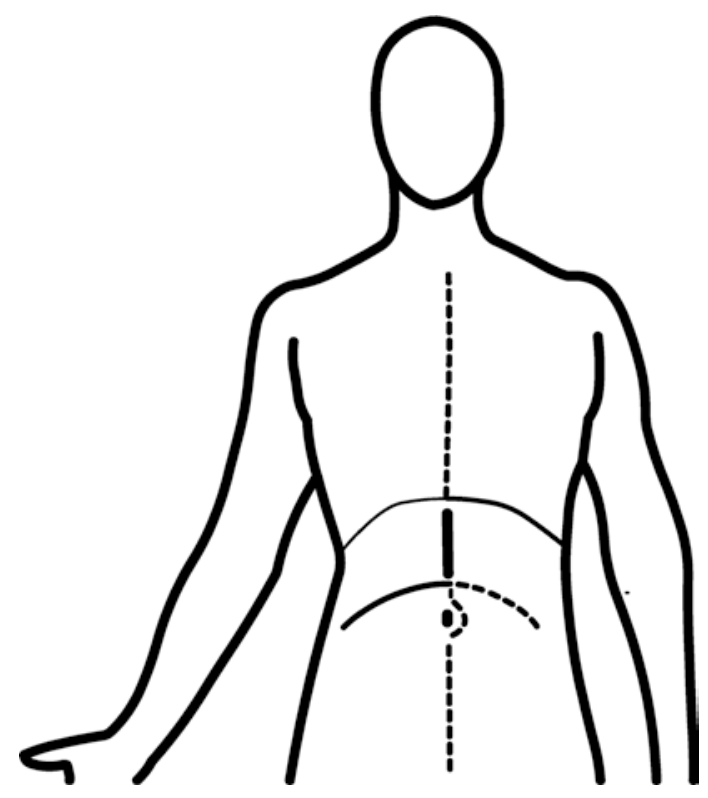

Fig. 32.10 Incision options. See text for description

cava from top view or from sides with side rotation of the operating table and the surgeon sitting on an operating room stool. This helps much in lateral and posterior dissection of tumors, a note on which will be given later.

\section{Midline With or Without Transverse Extension}

Obese patients whom their fat is extra-abdominal are far friendlier than what one would expect. The weight of the abdominal wall fat seems to make it to fall off to the sides offering an unexpected access from the midline incision only.

\section{Any of the Above 2 with a Median Sternotomy}

Any of the above 2 can have a median sternotomy (partial or complete) as an extension according to ease of access to suprahepatic cava keeping a low threshold to do so. Its merits supersede morbidities. Patients with narrow subcostal angle or those with a decubitus characterized by big anteroposterior diameters at the level of epigastrium will probably require it for suprahepatic control. Should one be in a position to do a suprahepatic cava control for other reason than a large neoplasm, he would find this readily accessible without a sternotomy. And probably he will judge 
the recommendation of sternotomy as unnecessary or overdoing. This does not apply when a big tumor is inside especially for rt.-sided upper quadrant tumors which pushes the liver cephalad preventing downward pull on the liver even when mobilized. This downward pull is pivotal in the access. In addition, the process of liver mobilization itself can be very difficult, time consuming, and bloody when tumors in these locations are accessed without a thoracoabdominal incision. The authors choose the sternotomy out of the thoracoabdominal incisions options for its more suitability for the task and its lower morbidity including pain control and lesser respiratory complications.

In conclusion, the midline supraumbilical component of the wound and the rt. transverse component are the most mandatory and extendable. Therefore, one of them is favored for initial exploration. Should more access be needed for exploration the other is to be added. At this point one can decide-according to the lower limit of tumor-whether to extend the midline downward or do a left transverse extension. The median sternotomy is the same in either case.

One should not extend the rt. transverse to the It. side until the lower limit is settled because this 1t. side extension is a giveaway of the chance to extend the midline caudally.

\subsubsection{Role of Table-Mounted Retraction and Magnification}

There has to be a serious reason to start this type of surgery without the presence of a tablemounted retractor of any form, at least present in the operating room. The commercially available ones are many. These include the Bookwalter, the Omni-Tract, and the Iron Intern. They can all serve the task. It just takes some exercise to get to know how to maximize the benefit from any of them in a particular surgery with a particular incision. The author believes that surgeons need to know how to literally exploit such instruments (Fig. 32.11).

There might be an exception for the abovementioned near mandation of the use of tablemounted retractors which in case of huge tumors

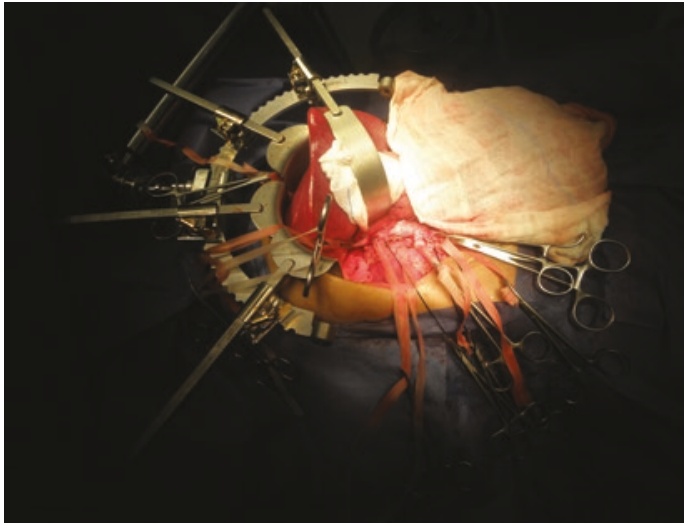

Fig. 32.11 The Bookwalter retractor in exposure of a vena cava harboring a tumor thrombus. From down the renal veins up to the intrahepatic vena cava, the field of surgery is exposed without a single hand of a human yet introduced. This frees the hands of the surgical team yet to come for some other tasks

stretch the abdominal wall. The latter falls off the tumor as soon as the abdomen is opened facilitating retraction. The choice of the tablemounted retractor and its positioning need to take into account the possible need for a sternotomy. They need to be placed from the beginning of surgery to allow this to happen swiftly when needed without disturbing the whole setup. One does not have to place them all away from the whole chest field though, for one will not be operating in the cephalic part of the chest. What is required is just a draping and positioning that allows the passage of the sternal saw to make the cut.

Combining the usage of two different tablemounted retraction systems might be some surgeon's choice, for example, a combination of a cable winch retractor for the costal margin and a Bookwalter or Omni-Tract for sides, caudal and visceral retraction. The cable winch retractor has a supreme performance in costal margin. As well, it offers ample space underneath to open the chest when needed. The Bookwalter and the OmniTract take on the side and caudal retraction as mentioned. It is therefore up to the surgeon and the availability of equipment.

One method for side retraction of abdominal wall is the use of the Finochietto chest wall retractor. This can be used instead of the standard 
Balfour retractor designed for the purpose. The Finochietto has a wider span and therefore suitable for midline incisions in subjects with big decubitus. The ones with fenestrated blades are favored as these fenestrations enable passing a stitch through them that anchors the blades to the abdominal wall to prevent its slippage from the abdominal wall edge.

As for magnification, though the cava is a gross structure and so is every structure in the field of vena cava-related tumors including the tumor itself, though this is the case, yet the author recommends the use of $2.5 \times$ magnifying loupe for most of the length of the surgery. Though structures are gross, yet it is not structures that one is after while using the loupes; one is after the tissue plans. The use of magnification helps much in defining tissue plans to desheath the cava and loop around the tributaries in proper planes with right angled forceps. During this looping one does not see the whole length of process of looping. The reliance here is on that the instrument has started in the proper subadventitial plan and therefore it is assumed that it will continue in it till the tip shows up again from the other side. This makes this proper start very important. Magnification is an important adjunct to this proper start.

\subsubsection{The Difference Between Lateral Dissection and Posterior Dissection}

Posterior dissection is complete when the tumor-cava complex is mobile over the vertebral column. The only way to do this is through opening of the inter-aortocaval plane with complete exposure of the aorta and ligation of lumbar arteries as necessary. It is complete only when a finger can be insinuated easily between the "tumor-cava complex" and the vertebral column all through the length of the tumor (Fig. 32.12).

When both aorta and cava are encased or in considerable contact with the tumor, it might be difficult to attain this step preserving both structures. For this "a cut to flip" technique can be introduced while handling the cava in which it is divided distally and flipped cranially to be taken

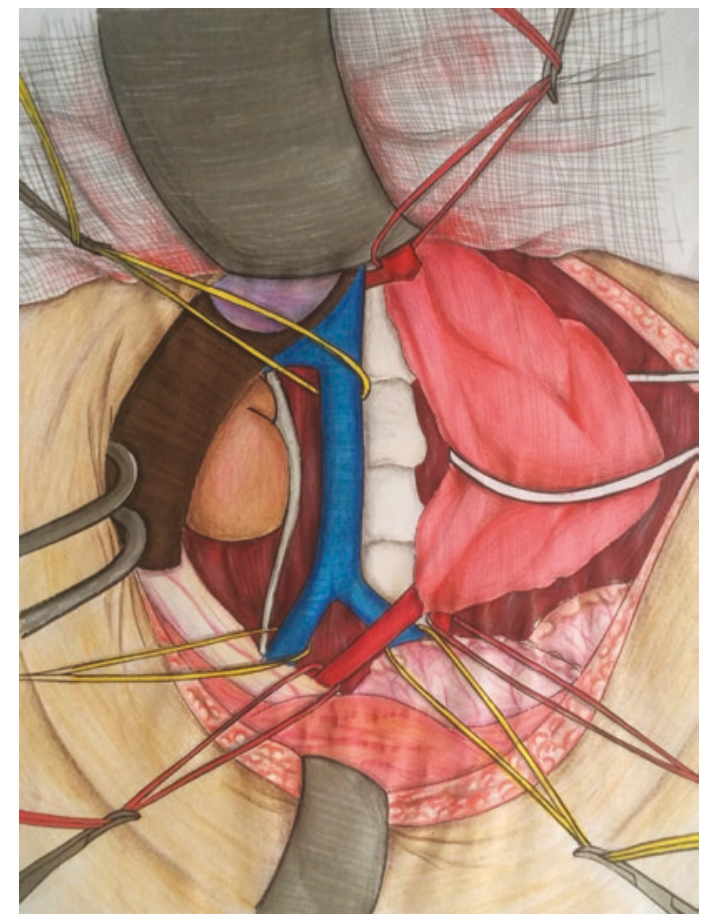

Fig. 32.12 Neuroblastoma encasing the aorta; demonstration of the so called completed posterior dissection as the aorta encased within a neuroblastoma is lifted over from the vertebral column completely

out of the field allowing a safer dissection lateral to and underneath the aorta (Fig. 32.13).

The aforementioned technique should be done with no hesitation. Merits far supersede cost in the given situations. Attempts to preserve both structures are a waste of time, blood, and effort of the patient and the team.

For tumors with adequate tissue planes, lateral dissection progresses seamlessly to the posterior dissection as the surgeon pulls on the tumor. Tumors with extensive desmoplastic reaction do not follow this. The lateral dissection is difficult and so is the posterior dissection. The lateral dissection does not take the surgeon seamlessly to the posterior one. In addition, they both will require too much sharp dissection and cautery. Tissues turn to sort of a "multilayered clothing" blending in with surroundings, and hence they require handling and cutting more or less like a parenchymatous organ harboring a tumor inside it that you cannot avoid cutting through it. 
Fig. 32.13 Cut to flip technique; (a) the IVC is transected caudally. (b) The resultant exposure and facilitation of posterior dissection and dissection from aorta. (c) Reanastomosis of the IVC
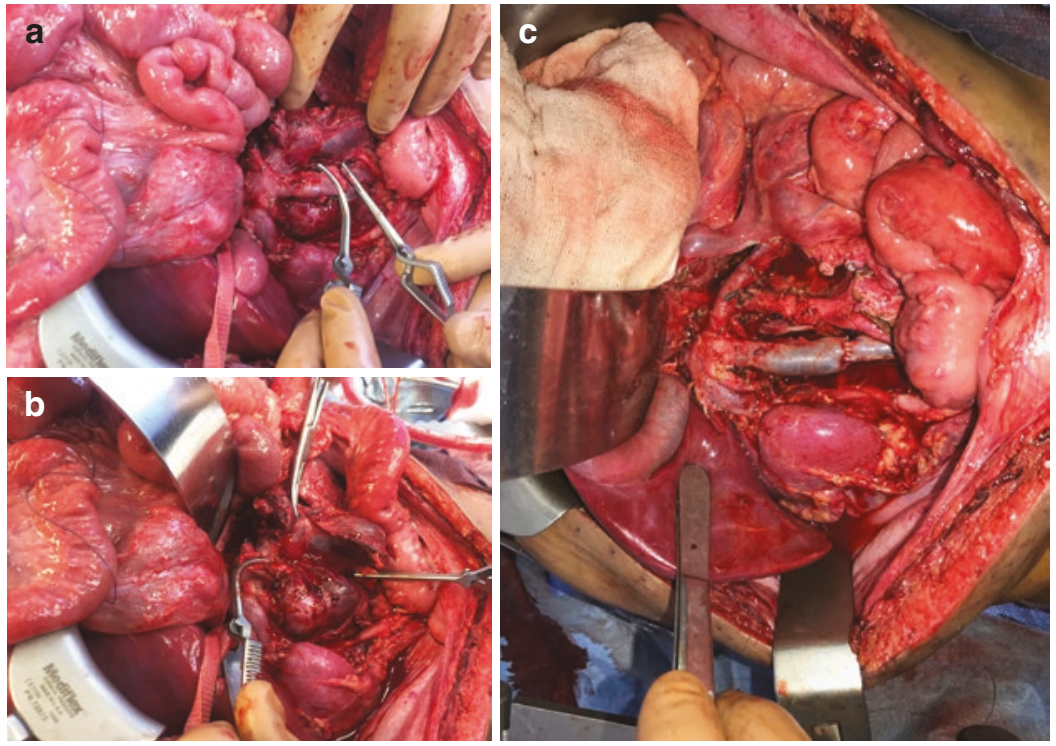

If the tumor is infiltrating the posterior abdominal wall laterally, i.e., the musculature not the vertebral column, here lateral dissection might be very bloody and requiring too much traction on the tumor risking their rupture which is a thing to be avoided at any cost. To avoid this, it is highly recommended to change the position of the patient from supine to lateral position while doing the lateral/posterior dissection. The time and effort consumed to do so is a very good investment indeed and should not be refrained from.

\subsubsection{Vena Cava Clamping}

Clamping of the vena cava can have some significant hemodynamic effects. An already compromised lumen should lessen such effects. Infrahepatic clamps are almost systematically tolerated without any specific preparation. Suprahepatic clamps on the contrary need special consideration. A test-clamp should be put on the proximal control and the operation is to be paused for a while. When the anesthetist reassures that the clamp is well tolerated in terms of hemodynamic effects, handling the cava can then start. If signs of hemodynamic instability are observed, the clamp is to be released until necessary measures are implemented, and the test is repeated.
Measures to enhance tolerance of this clamp are known to anesthetists. Before test clamping it might be helpful to put the operating table in a head-down position for few minutes in order to allow pooling of the blood in the upper half of the body.

Unless readily accessible and clear, it is recommended to defer the top control of the cava after the bottom control and the Pringle's maneuver. In case the vena cava is injured during dissection in this relatively tight space, there will be an opportunity to lessen the bleeding by crossclamping of the distal cava as well as occlusion of the Pringle's maneuver. If the repair can be done in these conditions, then well and good. If not, the hole is to be compressed and the liver returned back to rest on it. After which a sternotomy is to be performed and the intrapericardial cava is to be clamped. This is to be followed by returning back to the hole to get the necessary repair done.

Though total cross-clamps of suprahepatic vena cavas will eventually be tolerated by most if not all patients, it is to be mentioned that sometimes even the mere traction on the loopedaround hepatic veins might cause considerable hypotension. Should hypotension happen unexplainably during surgery, it is recommended to have a look at the slings looping round the 
hepatic veins. Even when they look innocent, they might need to be loosened completely or even removed, and the liver is to be returned back to the abdomen in the normal position.

\subsubsection{Vena Cava Grafting}

\section{Of the Threshold to Resect and Replace the Cava}

In short, cavas that are easily dissectable from the tumor are to be dissected and left in place.

Adherent cavas will require harsh manipulation. They are not expected to perform better than the graft after this harsh manipulation; they will probably occlude anyway. In this case if there is a technical advantage of removing the cava in terms of blood loss or access and safety of dissection of other more important structures, namely, aorta and its branches, the surgical team is then advised to keep a low threshold to cross-clamp the cava and resect it. Please see section for lateral and posterior dissection.

\section{Of the Best Caval Replacement Conduit}

There is a reasonable sum of literature on the topic from which one can come out with valuable information about this issue [1,2].

For small defects requiring patch graft, pericardial autografts serve as a good option as well as prosthetic grafts.

For full-circumference segment replacement, the body of literature present in the topic supports that PTFE is the conduit of choice. However, it is to be mentioned that not much Dacron grafts have been used for the purpose in the first place; those who have been used have performed as good.

In conclusion, if PTFE is available then it is reasonable to give it priority. However, if it is not available Dacron can be used instead.

The need for ring enhancement has been recommended by authors justified by the low pressure in the cava. However, it is to be remembered that the native cava is even of a more pliable wall than any graft. Therefore, ring-reinforced grafts are not to be considered a must unless for any reason the surgeon judges the presence of anatomical changes that will obviously compress the cava. An example of this is replacement of intrahepatic vena cava that required complete freeing from the central tendon.

\section{Of the Implantation of a Single Renal Vein in the Graft}

Caval resections above the level of renal veins would entail temporary interruption of renal vein and reimplantation into the graft (Fig. $32.8 \mathrm{~b}$ rt.). This interruption might take time and is liable to postoperative thrombosis.

Risk on the remaining kidney can be assessed by various methods (Table 32.5). Temporary occlusion while observing urine output and observing the kidney for color and turgidity changes indicates congestion. This can be supplemented by intraoperative Doppler and invasive pressure measurement of the occluded renal vein stump. The presence of high pressure indicates venous outflow obstruction. Not much of this situation is written about in literature to conclude the figures that indicates absolute safety of the kidney. If all parameters are normal after clamping, it is well understood that there should be no worry. Yet how far can we drift from normal in each parameter and still stay safe is yet to be determined in future work and publications. For now, the author recommends that if no change in the abovementioned parameters happens for $30 \mathrm{~min}$, then it is reasonable to assume that the kidney is having efficient collaterals. This is not very surprising as a tumor that requires resection of the cava must have narrowed its lumen and hence stimulating the collaterals development. If the parameters drift from normal, it will be left to the surgeon and radiologist to make a decision about the possible impact of this abnormality. It is recommended to have the Doppler done by a radiologist experienced in kidney transplant. His input will be based on experience from a close setting.

Table 32.5 Assessment of efficiency of collateral venous circulation

\footnotetext{
Assessment of efficiency of collateral venous circulation

1. Cross-clamping test and observing urine output, turgidity, and color

2. Doppler assessment during cross-clamping

3. Venous stump pressure
} 


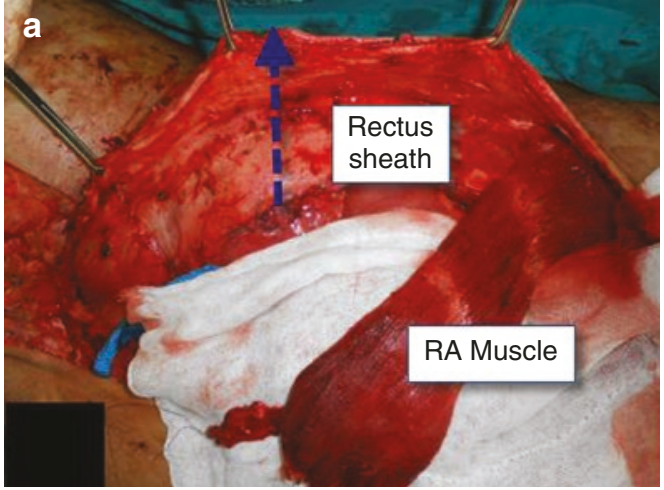

Fig. 32.14 (a) The rectus abdominis muscle (RA muscle) extracted from within the sheath before making the transverse extension of the wound marked by the blue dotted arrow. (b) Intra-abdominal placement of the muscle to cover suture lines. Here to cover the proximal

It is important to note that, while doing the assessment of efficiency of the collateral venous circulation of the kidney, the kidney is not to be mobilized or dissected for the purpose of better visualization. It should and it is always possible to do so while the kidney is still in place and not dissected. Mobilization and dissection can lead to disturbance of collaterals.

\section{Of the Need for Muscle Cover for the Repaired Cava}

The field after resection of these tumors is usually peritoneum-void leaving the repaired cava in contact with bowel. None of the authors who investigated the topic have reported the use of routine muscle cover. None reported entericcaval fistulas as well.

Based on the author's personal experience as well as others, perhaps it is reasonable to say that if there is no concomitant bowel anastomosis, then there is no need for routine muscle cover.

If there is one, then separation of the suture lines by omentum or muscle should be the rule unless it is very difficult to attain. The muscle of choice in this situation would be the rectus abdominis muscle superiorly based or inferiorly based according to the situation. This is important to plan ahead before the incision since bilateral transverse incision will cut through the

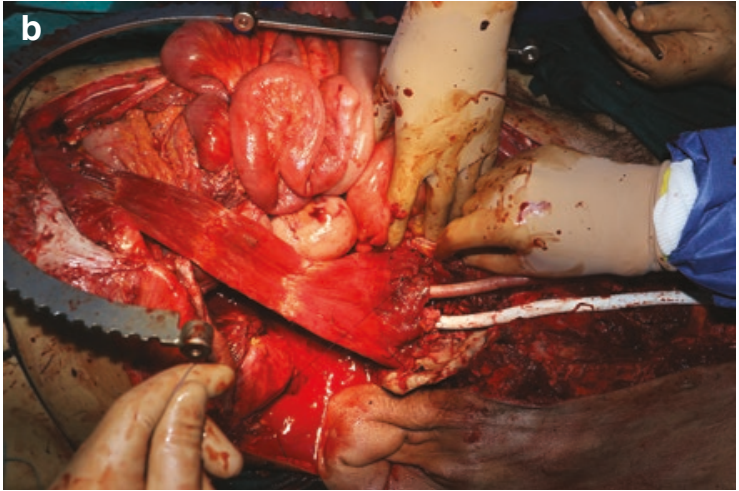

anastomosis of a PTFE graft to iliac vessels as well as an inferior vena cava suture line after resection of huge abdominal mass invading abdominal wall and extending to groin

muscle bilaterally. In this situation the procedure can start by raising the muscle from within the sheath before making the transverse cut (Fig. 32.14). Careful interpretation of the scans should inform about the need for bowel resection. Bowl resections away from caval suture lines need not be taken into consideration.

\subsubsection{Inviting an Old Technique: Vena Caval Compartmentalization}

Nothing in the surgeons' pocket to guard against thrombosis of the harshly treated cavas other than anticoagulation and hemodilution. The use of anticoagulation should be balanced against the risk of bleeding specially in the first postoperative day after a surgery with an extensive raw area.

Guarding against pulmonary embolism in a setting where anticoagulation might be contraindicated is a call for a vena caval filter. Placing a filter preoperatively requires awareness of its presence during surgery. Maneuvering the cava risks the dislodgement of the filter. If taking this caution is not preferred or if filters are not available, resorting to an old technique can be an alternative, which is compartmentalization of the cava by three to five polypropylene sutures $[3,4]$. Figure 32.15 demonstrates the technique. 


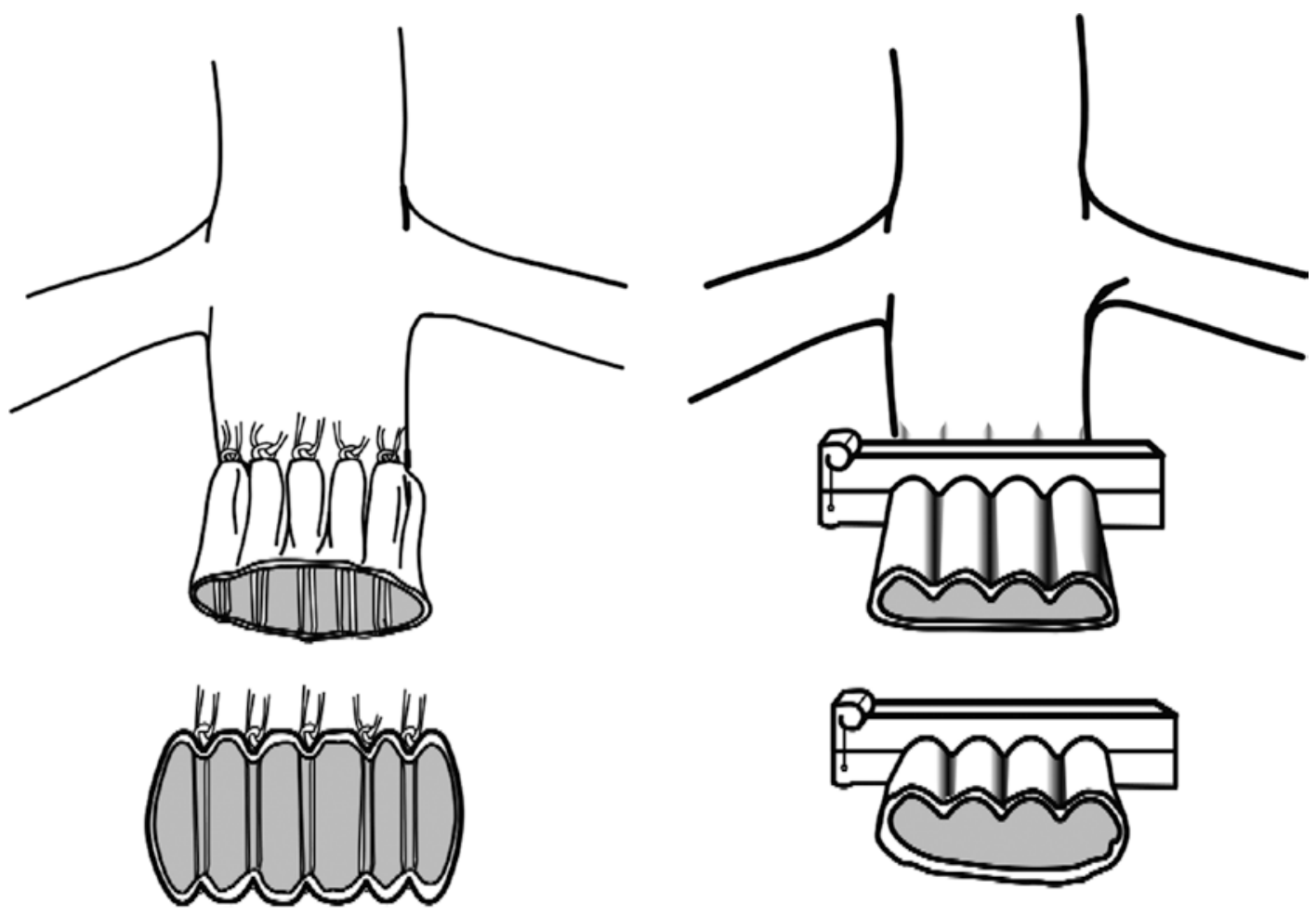

Fig. 32.15 Vena cava compartmentalization by plication technique using interrupted polypropylene sutures or by application of a specialized clip

\subsubsection{Adjunctive Techniques}

This section presents three adjunctive techniques that can be used. They are not basic components. Their place and indication are explained.

- Shunting and intraoperative balloon occlusion

- The use of cell savers

- In situ perfusion and ex vivo surgery

\subsubsection{Shunting and Intraoperative Balloon Occlusion}

Shunting the vena cava is a procedure that is usually required for trauma setting rather than for resection of tumors. Its application is particularly of usage for retrohepatic vena cava and intrahepatic vena cava. Shunting is not a maneuver to stop the bleeding. Vascular exclusion of the liver is the procedure that is intended for lessening of the bleeding; shunting is an adjunct to the vascular exclusion that allows flow from the lower body to reach the rt. atrium during the vascular exclusion. It is used when the vascular exclusion results in significant hypotension. It maintains a decent venous return for a patient who has already lost a significant amount of blood. The aforementioned scenario is not the usual case for vena cava-related tumor surgery. However, still it might happen. The options for shunting are veno-venous bypass or direct shunting by cuffed endotracheal tube of appropriate size $[5,6]$.

Balloon occlusion on the other hand is a method to stop bleeding. A Moore-Pilcher balloon is inserted through the femoral vein and advanced to the retrohepatic vena cava. Inflation of the balloon occludes the cava as well as the entry of the hepatic veins [5].

If inadvertent trauma to cava occurs or is expected, shunting might be still utilized in tumor surgery the same way it takes place in trauma.

In such a situation it is might be more accessible to introduce the shunt from a more caudal segment of the vena cava in a field with regular anatomy rather than from the rt. atrium. 
Table 32.6 Maneuvers to prevent air embolism in low pressure cavas

1. Direct manual sealing of the injury

2. Rush to the top control first before bottom control and porta hepatis control

3. Valsalva maneuver by anesthetist

4. Filling the field with saline solution with a rt. shoulder uptilt to prevent saline from pouring out

Table 32.7 Signs of low pressure in cava

1. Direct measurement of CVP reflects pressure in cava if the patient is lying flat

2. Absence of profuse bleeding from caval injury

3. Brisk venous pulsations in the vena cava

It is helpful to do the vascular exclusion in the following sequence: clamping the porta hepatis first and squeezing the liver manually to push the blood out to the hepatic veins followed by caval distal control and finally caval proximal suprahepatic control.

If the caval pressure is low, one should either do direct manual sealing of the injury, rush to the top control first before bottom control and porta hepatis control, perform Valsalva maneuver, or fill the field with saline solution all aiming at preventing air embolism (Table 32.6). Patients who do not bleed profusely from an injured cava are the ones of concern, for this indicates that the pressure in the cava is low, and therefore the possibility of sucking air is more likely. Table 32.7 lists signs of low caval pressure and therefore higher probability of air embolism.

\subsubsection{The Use of Cell Savers}

As is the case with surgery for any form of malignant disease, the use of cell savers is controversial. Investigators found that the probability of presence of malignant cells in the scavenged blood is relatively high $[7,8]$.

Two integral questions are still not completely answered: do these cells have the capacity to become metastases? If they do, is this risk more than the risks associated with allogenic blood transfusion? It is out of the scope of this text to discuss this literature in thoroughness. This has been tackled comprehensively in transfusion literature [9].
Since the issue is not finally resolved by transfusion specialists, surgeons therefore need to make their choice. What relates here is that in very few occasions the use of cell savers will be found necessary should the abovementioned strategies be entertained. It might be helpful to have it in the operating room for very special situations or for use after taking the tumor out. The use of cell savers while the tumor is still in is a matter of judgment and choice of the operating team which entails knowledge of the pathology, natural history of the disease being handled, and indeed the patient and his preferences. It will be a choice between permitting blood loss with its consequences-particularly the need for allogenic transfusion -in one side and the risk of permitting malignant cells of an unknown potential to get through to the blood stream.

\subsubsection{In Situ Perfusion and Ex Vivo Surgery}

Ex vivo resections are indicated for hepatic segment of vena cava. They entail complete extraction of the liver altogether with the IVC and indeed the tumor followed by bench surgery. This will require liver wash by an organ preservation solution (e.g., HTK solution) and cooling for the length of bench surgery. This technique has been more widely used and described by authors involved in management of Echinococcus granulosus infestation of the liver than in patients with malignancy in general. They reported liver bench time of $360 \mathrm{~min}$ well tolerated by the liver [10-12].

An alternative is in situ perfusion; in this technique only a caval segment is divided proximal and distal and the liver extruded from the abdomen yet the porta hepatis is left uncut and used-while in place-to flush the liver with preservation solution which is flushed out of the liver through the cut caval ends and sucked out of the field. It obtains a bloodless caval and liver dissection along with a better access to hepatic veins confluence area. After resection, these can be replanted in the native cava or to a graft according to the situation.

Ex vivo resections for tumoral surgery have been utilized by some authors with promising results [13]. 
The author would see ex vivo surgery as the future of cancer surgery not only to decrease blood loss and improve accessibility but also there might be an oncological advantage for this technique. Most of retroperitoneal resections entail dissection of important structures. Ex vivo surgery will enable these dissections to be done on the bench, and hence minimizing tumoral spillage should rupture or capsule breaches occur while trying to dissect intimate important structures.

\subsubsection{Special Situations}

- Off-pump excision of cavo-atrial junction thrombi

- Advocating staging and multiple sittings surgery: merits and philosophy of

\subsubsection{Off-Pump Excision of Cavo- atrial Junction Thrombi}

Cavo-atrial junction thrombus approached by heart bypass is widely described in the literature. "Vena cava freeing" technique described earlier can be applied up to this segment enabling such excisions to take place off-pump. This avoids cannulation and heparinization. This requires the following maneuvers (Fig. 32.16):

1. Division of the central tendon of the diaphragm longitudinally from sternum to few millimeters above the suprahepatic vena cava cut then encircles the cava leaving a cuff of central tendon on the caval wall.

2. This cut will lead the surgeon to the pericardial cavity seamlessly without much awareness of cutting the pericardium as a separate layer owing to the adherence of the pericardium to the central tendon in this area. The heart is protected from injury by cutting the central tendon-pericardium in the same manner that one cuts the peritoneum while entering the abdomen, pulling on a small bit between two artery forceps and making a small snip till entry takes place. This can be
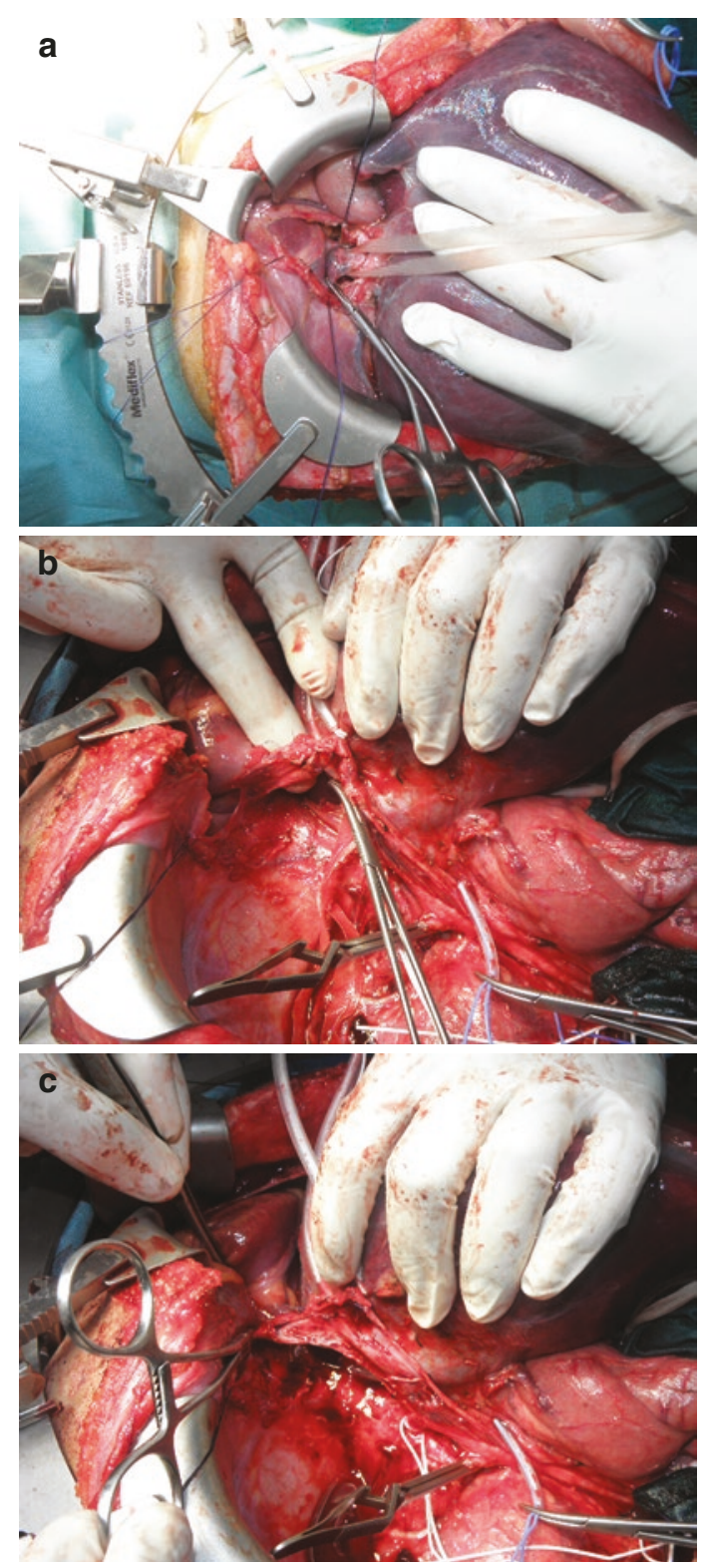

Fig. 32.16 (a) Exposure without sternotomy. Vena cava exposed yet not freed as necessary. (b) With sternotomy and vena cava completely freed denoted by the surgeon's finger passing behind the whole length of the suprahepatic cava from the heart to liver. (c) Vena cava clamped and opened. Note that freeing enabled placing the Satinsky clamp in a manner obtaining an extra centimeter in cranial direction by sliding cranially over the cavo-atrial junction now clamping on a bit of the atrial wall. This enabled rising over the top of the cavo-atrial junction thrombus off-pump 
started in the sternal side of the cut or even better halfway between the sternum and cava. The surgeon should be able to examine the span of the presumed cut and feel where the heart pokes the least while pulsating, and this spot is probably a favored spot for making the entry snip. The region near the cava is better avoided for the initial entry as the central tendon here fans and merges with cava and will make entry to the pericardial sac awkward.

3. Ligation of inferior phrenic vessels.

4. Freeing the cava posteriorly from the crus of the diaphragm (described earlier).

5. A low threshold to perform a sternotomy with or without manubriotomy.

\subsubsection{Advocating Staging and Multiple Sittings Surgery: Merits and Philosophy Of}

One thing about lengthy surgeries and surgeries that entails hemodynamic shifts is that they require a patient with a good physiological reserve. Several methods in medicine are in use to preoperatively assess the physiological reserve of different organ systems in a rather objective way.

In any surgery, patient's physiological status gets monitored minute by minute. The monitors in current practice offer you a panoramic view of this minute, yet they do not offer an eye on integral questions: "How much of the aforementioned physiological reserve is in action to offer the figures on the monitor? How much is still left in the reserves? For how long will they be able to do the required compensation to keep the physiological figures the way they are on the monitor?"

For instance, a mobile phone with a battery full will open up and do the call. When only $4 \%$ charge; it will still open up and do the call. Only when the call lasts long will the difference between the two shows up. One will continue and the other will shut down in the middle of the call.

We do not have the "\% of battery charge" display in any medical monitor, and therefore it is sometimes difficult to tell when will the physiological reserve "shut down." We only have our experience with these kinds of procedures corre- lated to some rough parameters like age range and preoperative assessment abovementioned. Simple factors like inadequate thermal control inside the O.R. can drain a patient's reserve far faster than expected.

Most of vascular and cardiac surgeries are "one go" surgeries in which you cannot but have it all in one sitting.

The case for resection surgery in oncovascular practice is different. The strategy abovementioned allows staging of the procedure in more than one sitting. It is an approach in which one can pull out in the middle of it-say after the first two phases - in one sitting and defer the others a week later. One other way of drawing the line between the sittings is the use a certain time interval for the sitting. A range of $4 \mathrm{~h}$ of anesthesia and operative time in these surgeries is a good landmark after which the wounds are closed in an extra hour and the patient transferred to the ward or ICU. The author recommends as well that the team takes a break in the middle of the sitting, an "obligatory" break in a sense.

By the author's direct observation, patients have been explored up to 10 days after the initial sitting, and the field was as exact as was left and even clearer. The concern about getting adhesions in such an interval is not true.

Add to this the value of giving the surgical team an opportunity to custom a more detailed plan to the second sitting after having the opportunity to acquire the givens learned in the first.

It is reasonable to assume that if the team is coming out of the surgery drained, then probably the patient is in jeopardy. An unnecessary one indeed, given the opportunity to stage the surgery. Loosing such an opportunity is such a waste.

There is nearly no cost paid for such staging. Proper information fed to the patient about this plan obviates the inconvenience of having to do two or three surgeries. It is all about anticipation. When anticipated it is well accepted.

It is not about doing "a heroic" surgery; it is about making "a heroic" surgery regular.

This has been the author's practice in multiple occasions. It has never been regretted. What has been regretted - in fact-is not doing so, when it has been listened to the monitors portraying that 
the patient's physiology is doing well and that the operation can carry on.

To make this clearer we all know that the adrenal gland is critical in stress. Its store of adrenaline and cortisol is key in these situations. As well, the liver glycogen is the source of energy. Current monitoring devices and techniques offers absolutely no idea about how much of these have been used and how much is left. This is a frontier to anesthesia and critical care medicine indeed.

In between the sessions, it is advisable to institute TPN. This should supply the building blocks for the reserves that one wishes to replenish.

Staging has been practiced and praised by different authors in similar situations $[14,15]$.

\subsection{Critical Note on Postoperative Care and Specific Complications}

\subsubsection{Anticoagulation}

Anticoagulation has been commented on earlier in the section "Inviting an old technique: Vena cava compartmentalization." Hemodilution and hydration are as relevant as anticoagulant drugs and should be sought with focus. Hematocrit is to be kept between 25 and 30\%. Blood transfusion should be kept to the minimum need.

\subsubsection{Monitoring}

The standard monitoring applies. Add to this a specific requirement.

For tumors that require dissection around the superior mesenteric artery (SMA) and in pediatric population who require "mobile evisceration" of the small bowel in the manner described, the occurrence of superior mesenteric artery thrombosis is a possibility. It is a deadly pathology and somehow silent. It does not necessarily reflect on clinical and vital signs with the gravity that calls for immediate exploration. Serum lactate has to be monitored closely with a low threshold for exploration. It is critical therefore to keep the patient volume replaced properly to avoid conflicting interpretation of high serum lactate and metabolic acidosis. These will commonly be attributed to general hypoperfusion. A well-perfused patient who still has acidosis and high lactate is one who is harboring a locally ischemic tissue somewhere in his body. The abdomen is a high-profile suspect.

When "mobile evisceration" of small bowel is utilized, it might be of value to stitch back the rt. colon to the paracolic gutter and the root of small bowel to retroperitoneum to prevent twisting over the SMA now acting as the only support.

The use of monitoring ileostomy (closed loop) for the first $48 \mathrm{~h}$ is to be considered in situations where SMA has been handled roughly.

It is left to reader to place these adjuncts as appropriate guided by (1) magnitude of bowel mobilization and SMA dissection and (2) efficiency of postoperative hemodynamic management and monitoring in each given setup.

This complication has been encountered by the author in pediatric population. The message is that the presence of a background disease in the vessel is not a necessity at all. Still in healthy vessels an eye has to be kept and the diagnosis entertained.

If a patient is diagnosed with the conditionspecially in pediatric population with small vessels-instead of accessing the SMA directly, it is recommended to access the SMA through a transverse aortic arteriotomy opposite to SMA takeoff. This is a convenient access for thrombectomy, flushing, and thrombolytic therapy injection, the repair of which is more accessible and less likely to invite thrombosis in itself (Fig. 32.17).

\subsection{Perioperative Mortalities and Oncological Outcome}

\subsubsection{Perioperative Mortalities}

The author's experience with the application of the abovementioned strategies and techniques is given here. These series are not all published to the moment. The results are given here to draw some light to the perioperative outcome. Sixtyfive patients in different centers have been 
Fig. 32.17 Access to SMA and SMA thrombectomy through transverse aortic arteriotomy opposite to SMA origin (shown by dotted line)

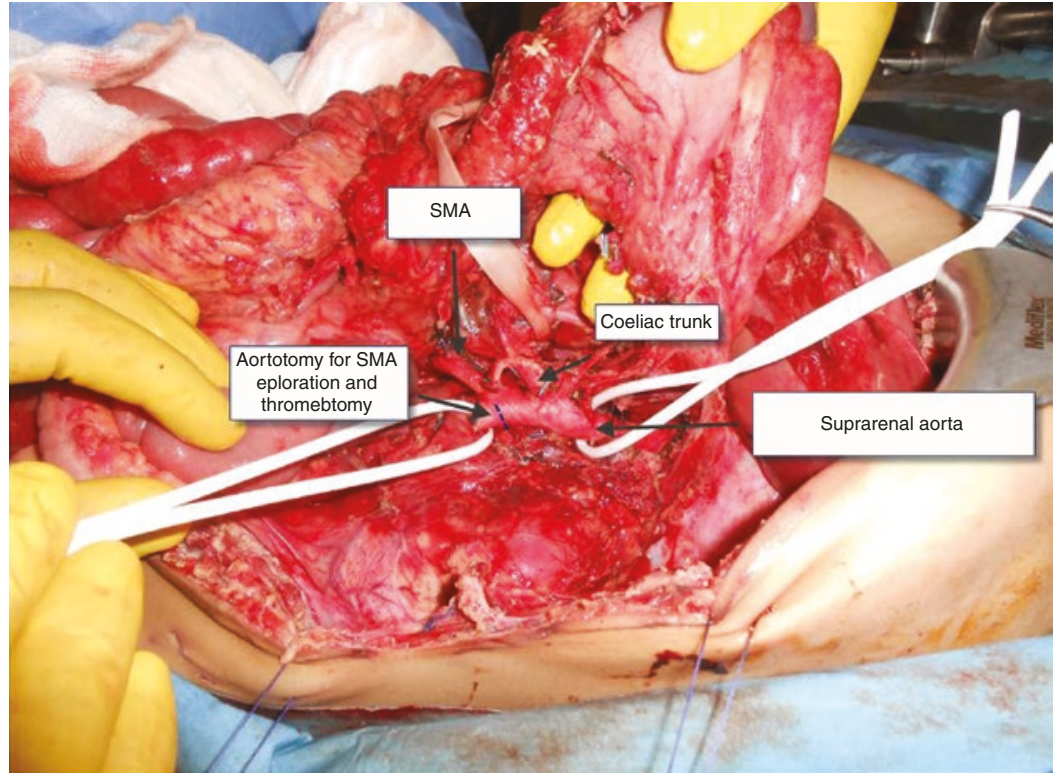

managed, 35 of which were vena caval thrombi and 30 had centrally located retroperitoneal tumors encasing either cava alone or cava and aorta. One of the former 35 died. Three of the latter 30 died perioperatively which makes a perioperative mortality rate of $6.1 \%$. Significant morbidities include a single patient with postoperative renal vein thrombosis and ATN that resolved with exploration, thrombectomy, and supportive medical treatment without the need for dialysis.

\subsubsection{Oncological Outcome}

Each of the case reports and series present in literature encompass a variety of tumors in terms of anatomical origin, stage, and histopathology [1, 2, 16-23]. It is not applicable therefore to release a survival rate for the entity "vena cava-related tumors." What brought them together is the need for application of a unified technical approach. Each of which is expected to follow-in terms of survival-the same figures that are expected for their counterparts of the same histopathology and tumor biology, etc.

For instance, a neuroblastoma encasing the cava will follow the same rules that govern survival in neuroblastoma in general which is, in this occasion, the risk stratification in common use putting into effect an interplay between five different factors that includes tumor stage, $\mathrm{N}$-myc amplification, and others.

Should the decision to operate or not is to be based on the expected oncological outcome; this will need to be made patient by patient with proper patient counseling.

This abovementioned concept has been put to test and investigated by authors in the field of pancreatic adenocarcinoma. Matched results have been found when patients who required arterial resection along with the pancreaticoduodenectomy are matched to retrospective controls who did not require vascular resection [24].

\subsection{Reproducing the Service and the Making of the Oncovascular Surgeons and Service}

In a dedicated future publication, the author intends to address this topic in detail. For now, a note on the basic concepts that will be introduced is going to be mentioned and these are: 
First: This is a hybrid technique between oncology, vascular, microvascular, and transplantation surgeries.

Second: The system proposed to produce these oncovascular surgeons is intended-contrary to presumption-not to be labor intensive or extended over a long duration. It is intended to be an economic model of surgical education and "training." Our current models are wasteful somehow, and it is to be admired that our medicine is still incomplete, and therefore we have to find a way to get people to learn it fast and economically to give them space, energy, and fund to look for real breakthroughs and look after their social life. That is what medicine is all about, to get people back to their social life healthy, isn't it? Medical practitioners are part of this.

\subsection{Summary}

- This is a unified approach to retroperitoneal tumors involving central structure in the abdomen, namely, cava, aorta, and their tributaries and branches.

- The procedure passes through four phases in a particular order.

- The phases put in this sequence allow staging, decrease blood loss, and enable management of different technical situations being it contact, infiltration, encasement, or intraluminal tumoral extension.

- Posterior dissection might require change of position of the patient.

- Posterior dissection might require cutting the cava, flipping it, and anastomosing it after resection.

- No need for cell savers in most cases.

- Tumors up to cavo-atrial junction can be removed off-pump.

- When liver resection is required, ex vivo resection or in situ perfusion can be used.

- One will never regret:

- Staging the procedure

- Bigger access, particularly sternotomy

- Caval resection and replacement
- It is not about doing a heroic surgery; it is about making a heroic surgery regular and reproducible in different setups.

- Three points are yet to be made sharp in the future research:

- How safe or unsafe are cell savers

- For now use to the minimum indications.

- Minimum parameters that indicates the efficiency of the collateral venous circulation of the kidney

- For now Doppler by a kidney transplant radiologist is the current best option.

- Oncological outcome for specific tumor types

- For now base decisions on the results of their counterparts in other anatomical locations.

Acknowledgments The author would like to thank Dr. Hussein Khairy for his integral role in the development of the author's oncovascular surgery practice.

Thanks to Mr. Mark Tyrell for his key contribution in providing the concept of "vena cava-related tumors" and for his pushing forward to publish it.

Thanks to Mr. Morad Sallam for the time he offered providing opinion, liaising, and facilitating the publication.

Thanks to Mr. Mohamed Shahm, Mrs. Heba Eldeeb and Ms. Anna Boriak for their efforts doing the diagrammatic representation.

\section{References}

1. Hardwigsen J, Baque P, Crespy B, Moutardier V, Delpero JR, Le Treut YP. Resection of the inferior vena cava for neoplasms with or without prosthetic replacement. Ann Surg. 2000;233(2):242-9.

2. Silva E, Pedro LM, Moutinho M, Amorim P, Evangelista A, Ortiz S, López D, e Fernandes JF. Leiomyosarcoma of level II inferior vena cava; an original solution for bilateral renal vein reconstruction. Angiol Cir Vasc. 2016;12(1):55-61.

3. Eklof BGH. Deep venous thrombosis and pulmonary embolism. In: Chant ADB, Barros D'Sa AAB, editors. Emergency vascular practice. London: Arnold; 1997. p. $135-50$.

4. Criado E, Johnson G Jr. Prevention of pulmonary emboli. In: Jamieson CW, Yao JST, editors. Rob and Smith's operative surgery; vascular surgery. London: Chapman and Hall Medical; 1994. p. 593-600.

5. Burch JM, Franciose RJ, Moore EE. Trauma. In: Schwartz SI, Tom Shires G, Spencer FC, Daly JM, 
Fischer JE, Galloway AC, editors. Principles of surgery. Singapore: McGraw Hill; 1999. p. 190.

6. Demetriades D. Abdominal vascular injuries. In: Rutherford RB, Cronenwett JL, Glovicizki P, Wayne Johnston K, Krupski WC, et al., editors. Rutherford vascular surgery. Philadelphia: Elsevier Saunders; 2005. p. 1040-2.

7. Waters JH, Donnenberg AD. Blood salvage and cancer surgery: should we do it? Transfusion. 2009;49(10):2016-8.

8. Catling S, Williams S, Freites O, Rees M, Davies C, Hopkins L. Use of leucocyte filter to remove tumour cells from intraoperative cell salvage blood. Anaesthesia. 2008;63:1332-8. [PubMed: 19032302] 15.

9. Hansen E, Wolff N, Knuechel R, Ruschoff J, Hofstaedter F, Taeger K. Tumor cells in blood shed from the surgical field. Arch Surg. 1995;130:387-93.

10. Wen H, Dong J-H, Zhang J-H, Zhao J-M, Shao Y-M, Duan W-D, Liang Y-R, Ji X-W, Tai Q-W, Tuerganali A, Li T. Ex vivo liver resection followed by autotransplantation for end-stage hepatic alveolar echinococcosis. Chin Med J. 2011;124(18):2813-2817 2813.

11. Jianyong L, Jingcheng H, Wentao W, Lunan Y, Jichun Z, Bing H, Ding Y. Ex vivo liver resection followed by autotransplantation to a patient with advanced alveolar echinococcosis with a replacement of the retrohepatic inferior vena cava using autogenous vein grafting: a case report and literature review. Medicine (Baltimore). 2015;94(7):e514.

12. Hemming AW, Chari RS, Cattral MS. Ex vivo liver resections. Can J Surg. 2000;43(3):222-4.

13. Kato T, Lobritto SJ, Tzakis A, Raveh Y, Sandoval PR, Martinez M, Granowetter L, Armas A, Brown RS Jr, Emond J. Multivisceral ex vivo surgery for tumors involving celiac and superior mesenteric arteries. Am J Transplant. 2012;12(5):1323-8.

14. Zhang A-Q, Xue M, Wang D-J, Nie W-P, Xu D-B, Guan X-M. Two-stage resection of a disseminated mixed endometrial stromal sarcoma and smooth muscle tumor with intravascular and intracardiac extension. Taiwan J Obstet Gynecol. 2015;54:776-9.

15. Mohd Ariff S, Joehaimey J, Ahmad Sabri O, Abdul Halim Y. Two-stage surgery for a large cervical dumb- bell tumourin neurofibromatosis 1: a case report. Malays Orthop J. 2011;5(3):24-7.

16. Vladov NN, Mihaylov VI, Belev NV, Mutafchiiski VM, Takorov IR, Sergeev SK, Odisseeva EH. Resection and reconstruction of the inferior vena cava for neoplasms. World J Gastrointest Surg. 2012;4(4):96-101.

17. Kobayashi T, Miura K, Ishikawa H, Soma D, Zhang Z, Yuza K, Hirose Y, Takizawa K, Nagahashi M, Sakata J, Kameyama H, Kosugi S, Wakai T. Successful reresection for locally recurrent retroperitoneal liposarcoma at four years after ex vivo tumor resection and autotransplantation of the liver: a case report. Transplant Proc. 2016;48:1215-7.

18. Berumen J, Hemming A. Vascular reconstruction in hepatic malignancy. Surg Clin N Am. 2016;96: 283-98.

19. Goto H, Hashimoto M, Akamatsu D, Shimizu T, Miyama N, Tsuchida K, Tajima Y, Ohuchi N. Surgical resection and inferior vena cava reconstruction for treatment of the malignant tumor: technical success and outcomes. Ann Vasc Dis. 2014;7(2):120-6.

20. Castelli P, Caronno R, Piffaretti G, Tozzi M, Lomazzi C, Dionigi G, Boni L, Dionigi R. Surgical treatment of malignant involvement of the inferior vena cava. Int Semin Surg Oncol. 2006;3:19.

21. Sarkar R, Eilber FR, Gelabert HA, Quinones-Baldrich WJ. Prosthetic replacement of the inferior vena cava for malignancy. J Vasc Surg. 1998;28:75-83.

22. Bower TC, Nagorney DM, Cherry KJ Jr, Toomey BJ, Hallett JW, Panneton JM, Gloviczki P. Replacement of the inferior vena cava for malignancy: an update. J Vasc Surg. 2000;31:270-81.

23. Shen Z-J, Zhou X-L, Yu Y-L, Li M. One case of leiomyosarcoma of the inferior vena cava treated with radical resection and vascular reconstruction. Vasc Med. 2005;10:225-7.

24. Bachellier P, Rosso E, Lucescu I, Oussoultzoglou E, Tracey J, Pessaux P, Ferreira N, Jaeck D. Is the need for an arterial resection a contraindication to pancreatic resection for locally advanced pancreatic adenocarcinoma? A case-matched controlled study. J Surg Oncol. 2011;103(1):75-84. 